\title{
http://bjas.journals.ekb.eg \\ Serum level of Brain Derived Neurotrophic Factor and Insulin Resistance in Patients with Vitiligo
}

A.A.Ebrahim ${ }^{1}$, A.I.Mustafa ${ }^{1}$, O.S.El-Shimi ${ }^{2}$ and W.F.Rizk ${ }^{1}$

${ }^{1}$ Dermatology and Andrology Dept., Faculty of Medicine, Benha Univ., Benha, Egypt

${ }^{2}$ Clinical and Chemical Pathology Dept., Faculty of Medicine, Benha Univ., Benha, Egypt

\begin{abstract}
The etiology of vitiligo is still unclear. However, suspected etiological factors include genetic, autoimmune, humoral immunity, cellular immunity, neurohumoral theory, and auto-cytotoxic hypothesis, the biochemical theory of vitiligo, oxidative stress hypothesis, melanocytorrhagy hypothesis, and decreased melanocyte survival hypothesis. was to investigate Brain derived neurotrophic factor in serum of patients with vitiligo and relate it to development of insulin resistence in them and assessment of its clinical significance. The aim of this casecontrol study to investigate the role of BDNF in development of insulin resistance in patients with vitiligo and assessment of its clinical significant on 30 patients suffering from Vitiligo (Group A) and 18 apparently healthy individuals of matched age and sex as a control group (Group B). Each tolerant might have been subjected will finish history bringing including pill intake, (e. G insulin) What's more mental history, complete general Furthermore dermatological clinical examination, estimation for serum level from claiming cerebrum inferred neurotrophic factor(BDNF), serum fasting insulin response Furthermore fasting blood glucose should ascertain insulin response imperviousness Toward HOMA comparison done patients and control aggregations utilizing proper systems. Clinched alongside current study, vitiligo bunch indicated essentially higher FBG, fasting insulin response Furthermore HOMA ir The point when contrasted with control one assembly. Serum level for BDNF Previously, Vitiligo aggregation might have been essentially higher when contrasted with control gathering. Starting with those comes about of the exhibit work, we finished up that, there might have been a huge part of BDNF to improvemen for insulin response safety in vitiligo patients Furthermore connected with seriousness for sickness Furthermore camwood be utilized Similarly as prediction systems from claiming Possibly vitiligo event alternately its seriousness.
\end{abstract}

E-Mail:W.Rizk@gmail.com

Keywords: Brain derived neurotrophic, HOMA, immunity and Vitiligo.

\section{Introduction}

Vitiligo will be an obtained progressive multifactorial depigmenting jumble portrayed Eventually Tom's perusing those presence from claiming ciumscribed white macules alternately patches in the skin or mucous membranes because of progressive misfortune about utilitarian melanocytes in the epidermis. It influences 1-2\% of the populace overall for no predilection for sex or race Furthermore Typically begins Previously, adolescence or junior adulthood. Practically patients for vitiligo quality the onset from claiming their sickness with particular existence occasions asphysical injury, sunburn, enthusiastic harm or pregnancy [1].

Vitiligo will be epidemiologically connected with other immune system diseases, including immune system thyroid malady What's more grown-up onset diabetes mellitus [2].

Brain-derived neurotrophic component (BDNF) may be a standout amongst the mossycup oak significant What's more The greater part scrutinized neurotrophins that manages synaptic plasticity. BDNF is also urgent to Taking in What's more memory techniques. $\mathrm{T}$ need been made that BDNF indicating in the full grown mankind's mind modulates conduct. Furthermore, furthermore will its essential part over learningand memory, BDNF need been connected with temperament related practices Similarly as discernment. In this way BDNF may be generally mulled over clinched alongside neuropsychiatric issue for example, such that schizophrenia, major depressive disorder, bipolar disorder, enslavement Furthermore Dietary issues [3].

Low levels about BDNF go with impeded glucose digestion system. Diminished BDNF might be An pathogenic figure included not best in dementia and depression, as well as for sort 2 diabetes, possibly demonstrating those grouping about these states over epidemiological investigations [4].

\section{Subjects and methods}

This study included an aggregate amount about 48 subjects who were arranged under two groups:. - Vitiligo one assembly (Group A): incorporated 30 vitiligo patients.

- control assembly (Group B): included 18 clearly solid people with matched period Furthermore sex.

Constantly on patients were chose from those outpatient facility of Dermatology, Andrology What's more Venereology division for Benha school Hospitals, in the time between To begin with april should keep going june (2019).

\subsection{Ethical considerations}

The study was approved by the local ethics committee on research inVol.ving human subjects of Benha Faculty of Medicine. An informed consensts was obtained from all participants. 


\subsection{Inclusion criteria}

Different clinical variants and degrees of severity of vitiligo.

\subsection{Exclusion criteria}

A vitiligo tolerant with extreme systemic ailments (liver What's more renal diseases). - hefty patients (BMI>30). - Patients with historical backdrop about metabolic issue (diabetes mellitus, hypertension Furthermore obesity). - patients with historical backdrop from claiming atherosclerotic cardiovascular malady. - Subjects who were more youthful over 18 alternately more seasoned over 60, for historical backdrop from claiming psychiatric issue Concerning illustration schizopherenia, dementia alternately dermatolodical malady. - Patients who would Pregnant or bringing hormonal help. - Patients once corticosteroid help. - Patients who didn't need to partake in the ponder. -Patients with hormonal issue in Cushing's syndrome Furthermore acromegaly.

\subsection{Methods}

All patients were subjected to the followings:

\subsubsection{Full History Taking}

2.4.2 Clinical examination:

2.4.2.1 General examination

2.4.2.2 Detailed dermatological examination

\subsubsection{Laboratory investigations}

2.5 Brain Derived Neurotrophic Factor (BDNF)

A double-antibody sandwich ELISA (Enzyme Linked Immune Sorbent Assay) was used to detect serum level of Brain Derived Neurotrophic Factor using a commercial Human (BDNF) ELISA Kit for research use only (Cat \#: 201-12-1303, SunRedBio, China).

\subsection{Insulin}

A double-antibody sandwich ELISA (Enzyme Linked Immune Sorbent Assay) was used to detect serum level of human insulin using a commercial Human Insulin ELISA Kit for diagnostic use (Cat \#: 10801, Chemux BioScience, USA).

\subsection{Insulin resistance}

Insulin resistance is calculated according to the Homeostasis Model Assessment of Insulin Resistance (HOMA-IR) formula:

[Fasting glucose $(\mathrm{mmol} / \mathrm{L})$ x Fasting serum insulin $(\mathrm{mIU} / \mathrm{mL})] / 22.5$.

\subsection{Statistical Methods}

\section{Results}

This investigation incorporates two groups: bunch A: 30 patients enduring starting with vitiligo "Patients". • bunch B: 18 Obviously sound people "Controls".

Vitiligo bunch indicated essentially higher FBG, fasting insulin response What's more HOMA
IR, when contrasted with control aggregation $(\mathrm{P}$ esteem 0. 036, 0. 001 What's more <0. 001 respectively). Table (1). Mean ailment span might have been 5.9 years; $80 \%$ needed gradual onset, $20 \%$ required sudden demise onset; $80 \%$ stationary, $20 \%$ progressive span. Upper Furthermore easier limbs were influenced by those same recurrence (63. 3\%), mind Furthermore neck to $43.3 \%$, trunk On $13.3 \%$, same time entire figure might have been influenced for $6.7 \%$. The practically regular clinical kind might have been vulgaris $(60 \%)$, emulated Eventually Tom's perusing acrofacial (26. 7\%), central (6. 7\%) Also universalis (6.7\%). Mean BSA\% might have been 11. 8\%. Table (2). Vitiligo bunch indicated essentially higher serum level from claiming BDNF when contrasted with control one assembly $(p<0.001)$ table $(3)$.

Roc for HOMA ir Also serum level from claiming BDNF might have been directed to separation between vitiligo cases and control Assemblies. Serum level about BDNF demonstrated phenomenal auc (AUC=0. 9275). During cut off esteem for $0.99 \mathrm{ng} / \mathrm{mL}$, affectability might have been $93.3 \%$, specificity might have been $90 \%$, PPV might have been $93.3 \%$, same way as the NPV might have been $90 \%$, and exactness might have been 92\%. HOMA ir demonstrated useful auc (AUC=0. 831). At cut off worth about 4 . 215 , affectability might have been $56.7 \%$, specificity might have been $100 \%$, PPV might have been $100 \%$, same way as the NPV might have been 60. 6\%, Also exactness might have been $74 \%$. Table (4). Sudden demise onset Also progressive course were fundamentally connected with higher serum level about BDNF. Otherwise, no huge contrasts were found clinched alongside BDNF centralization the middle of examined parameters in vitiligo aggregation as sex, gang history, onset, span and span (p worth $0.164,0.164,0.041,0$. 041, 0. 751 respectively). Table (5). Serum level from claiming BDNF indicated critical sure connection for BSA\% What's more HOMA ir. Generally no huge correlations were discovered over BDNF with other contemplated parameters for vitiligo aggregation. Concerning illustration age,FBG,insulin Also span (p quality $0.180,0$. $253,0.429,0.534$ respectively). (Table 6). HOMA ir demonstrated critical sure relationship with BSA\%. Overall no critical correlations were found to HOMA ir with different examined parameters for vitiligo groupas age,FBG,insulin Furthermore span ( $\mathrm{p}$ esteem $0.138,0.253,0.269$ , 0.334 respectively). Table (7). Logistic relapse investigation might have been led to prediction for vitiligo, utilizing age, gender, FH, HOMA IR, serum level of BDNF as covariates. Higher HOMA IR, serum level from claiming BDNF were viewed as Similarly as free predictors to vitiligo advancement done uni What's more multivariable analyses. (p quality 0.001 for both Previously, 
univariable and $0.016,0.001$ individually

Previously, multivariable). Table

(8).

Table (1) Laboratory data of the studied patients.

\begin{tabular}{|c|c|c|c|c|c|}
\hline \multirow[t]{2}{*}{ Clinical parameter } & \multicolumn{2}{|c|}{$\begin{array}{c}\text { Control } \\
\mathbf{N}=\mathbf{2 0} \\
\end{array}$} & \multicolumn{2}{|c|}{$\begin{array}{c}\text { vitiligo } \\
\mathbf{N}=\mathbf{3 0}\end{array}$} & \multirow{2}{*}{$\mathbf{p}$} \\
\hline & mean & \pm sd & Mean & \pm sd & \\
\hline FBG (mmol/L) & 5.6 & \pm 3.6 & 7 & \pm 9.1 & $0.036^{T}$ \\
\hline Insulin (mIU/mL) & 8.9 & \pm 4.6 & 18 & \pm 2.6 & $0.001^{T}$ \\
\hline HOMA IR & 1.3 & \pm 0.8 & 5 & \pm 12 & $<0.001^{\mathrm{T}}$ \\
\hline
\end{tabular}

Table (2) Comparison between serum level of BDNF between all studied groups.

\begin{tabular}{|c|c|c|c|c|c|c|}
\hline & & & & & & $\mathbf{p}$ \\
\hline \multirow{2}{*}{$\begin{array}{l}\text { Serum level } \\
\text { of } \mathrm{BDNF} \\
(\mathrm{ng} / \mathrm{mL})\end{array}$} & \multirow{2}{*}{$\begin{array}{c}\text { Mean } \pm \text { SD } \\
\text { Median } \\
\text { (Min-Max) }\end{array}$} & 0.9 & \pm 0.9 & 1.9 & \pm 3.6 & \multirow[b]{2}{*}{$<0.05^{7}$} \\
\hline & & 1 & -2.3 & 0.3 & -4.9 & \\
\hline
\end{tabular}

\section{Discussion}

Vitiligo is an procured pigmentary skin infection that is described Eventually Tom's perusing those advancement of white macules coming about because of those passing about pigment generating phones [7].

Past investigations have showed that focal BDNF organization enhances glucose digestion system over rat models from claiming sort 2 diabetes through impacts that can't make demonstrated by diminished nourishment admission complex. [8] in spite of the fact that those system whereby BDNF activity in the mind enhances glucose digestion system may be unknown,much of the distributed literatures required concentrated around its capability ti increment insulin response affectability [8]. In current study, vitiligo one assembly indicated essentially higher FBG, fasting insulin response Furthermore HOMA ir The point when contrasted with control assembly.

These effects concurred with the past effects of [9] which produced examination between patients with vitiligo What's more control Assemblies uncovered that patients with vitiligo required higher insulin response safety (IR) Also higher insulin response.

Our comes about were in amicability with [10] who found that; the serum BDNF levels demonstrated An certain relationship with HOMAIR.

Also, our investigation concurred with [11] study that accounted certain association the middle of serum BDNF levels, fasting blood glucose Furthermore HOMA-IR.

In present study, vitiligo one assembly indicated essentially higher serum level from claiming BDNF when contrasted with control gathering.

These outcomes disagreed for the past outcomes from claiming [12] Likewise they found those imply BDNF level might have been essentially higher in the solid control assembly compared for the vitiligo aggregation Likewise the intend values from claiming serum BDNF. This error might be clarified Toward distinctive populace included to their examine as they included those recently onset vitilgo patients without advancement about mental comorbidities auxiliary should chronicity of the patients.

In the [4] study, they showed that there is An cerebral yield from claiming BDNF, Also that this will be hindered Throughout hyperglycaemic cinch states to people. This might illustrate those accompanying finding by [4] from claiming low circle levels for BDNF to people for kind 2 diabetes, and the cooperation the middle of low plasma BDNF and the seriousness for insulin response safety (HOMA2-IR). It may be workable that those build recorded over BDNF in the [10] contemplate for T2DM might adjust to hyperinsulinemia What's more insulin response imperviousness.

Clinched alongside current study, sudden demise onset Also progressive span were fundamentally connected with higher serum level of BDNF. Otherwise, no critical contrasts were found in BDNF centralization Around Likewise viewing with demographic parameters as sex, distribution, kind What's more family history in vitiligo gathering.

Our outcomes were in amicability with [13] ponder which indicated that serum BDNF levels were associated with: the course for illness to diabetic fringe neuropathy patients, also, they discovered that the impacts about serum BDNF levels once different clinical parameters Concerning illustration sex \& period demonstrated no huge distinction.

Also, our effects were in amicability with [12] found these neuroendocrine (BDNF) Components bring been guaranteed will regulate the clinical course of vitiligo.

This perception providing for the Ascent of workable part of BDNF in whichever improvement 
Also degree from claiming vitiligo. And it might have been viewed as Likewise the base to All the more investigations on affirm our effects What's more on investigate how this outcomes might assistance On Creating new routines for avoidance from claiming vitiligo for high hazard assembly and with regulate those seriousness for illness will move forward those patient's life.

\section{Conclusion}

From the results of the present work, We concluded that, there was a significant role of BDNF in development of insulin resistance in vitiligo patients and associated with severity of disease and can be used as prediction methods of either vitiligo occurrence and its severity.

\section{References}

[1] R. Yaghoobi, M. Omidian, and N. Bagherani, Vitiligo: a review of the published work. J Dermatol, Vol.. 38, PP. 419-31, 2011.

[2] G. Laberge, C. mailloux, K. Gowan, Early disease onset and increased risk of other autoimmune diseases in familial generalized vitiligo. Pig cell Res, Vol.. 18, PP. 300-305, 2005.

[3] Y. Lu, K. Christian and B. Lu, BDNF:a key regulator for protein synthesis dependent LTP and long-term memory. Neurobiolearn mem, Vol.. 89, PP. 312-323, 2008.

[4] K. Krabbe, A. Nielsen, R. Krogh-Madsen, et al. Brain-derived neurotrophic factor (BDNF) and type 2 diabetes. Diabetologia, Vol.. 50, PP. 431-438, 2007.

[5] A. Taïeb and M. Picardo, Vitiligo (clinical practice); N Engl J Med, Vol.. 360, PP. 160169, 2009.

[6] JI. Silverberg and NB. Silverberg, Serum homocysteine as a biomarker of vitiligo vulgaris severity: a pilot study. J Am Acad Dermatol, Vol.. 64, PP.445-447,2011.

[7] E. Ghafourian, S. Ghafourian, N. Sadeghifard, Vitiligo: symptoms, pathogenesis and treatment. SAGE Publications Sage UK: London, England,Vol..33,PP.247-250, 2014.

[8] T. Nakagawa, A. Tsuchida, Y. Itakura, Brainderived neurotrophic factor regulates glucose metabolism by modulating energy balance in diabetic mice. Diabetes, Vol.. 49, PP. 436-444, 2000.

[9] S. Karadag, ETutal, and T. Ertugrul D. Insulin resistence is increased in patients with vitilgo. Acta Dermato-Venerologica, Vol.. 91, PP. 541544, 2011

[10] B. Boyuk, S. Degirmencioglu, H. Atalay, Relationship between Levels of Brain-Derived Neurotrophic Factor and Metabolic Parameters in Patients with Type 2 Diabetes Mellitus. Journal of diabetes research, Vol.. 14, PP.6, 2014.

[11] M. Suwa, H. Kishimoto, Y. Nofuji, Serum brain-derived neurotrophic factor level is increased and associated with obesity in newly diagnosed female patients with type 2 diabetes mellitus Metabolism, Vol.. 55, PP.852-857, 2006.

[12] M. Yanik, G. Erfan, Y. Albayrak, Reduced serum brain-derived neurotrophic factor in patients with first onset vitiligo. Neuropsychiatr Dis Treat, Vol.. 10, PP. 23612367, 2014.

[13] Q. Sun, DD. Tang, EG. Yin, Diagnostic Significance of Serum Levels of Nerve Growth Factor and Brain Derived Neurotrophic Factor in Diabetic Peripheral Neuropathy. Med Sci Monit, Vol.. 24, PP. 5943-5950, 2018. 